Check for updates

Cite this: Mater. Horiz., 2021, 8,1346

\section{Introducing the new Materials Horizons Advisory Board}

DOI: $10.1039 / \mathrm{d} 1 \mathrm{mh} 90021 \mathrm{~h}$

rsc.li/materials-horizons

This year, we have grown the Advisory Board for Materials Horizons in order to better represent the ever-increasing diversity of its authors and readers.
We feel that this group of active researchers, who have been carefully selected by our Editorial Board, will provide us with the valuable feedback and insights needed to continue the success of the journal in future. A table outlining members of the Advisory Board is shown below.
Markus Antonietti

David Beljonne

Chris Bettinger

Kanishika Biswas

Paul Blom

Mischa Bonn

Jean-Luc Brédas

Markus Buehler

Jillian Buriak

Yong Cao

Moyuan Cao

Rachel Caruso

Anthony Cheetham

Hong Chen

Brandi Cossairt

Dibyendu Das

Luisa De Cola

Ulrike Diebold

Mircea Dinca

Gitti Frey

Richard Friend

Subi George

Jian Ping Gong

Grace Gu

David Haddleton

Martin Heeney

Jurriaan Huskens

Hiroshi Imahori

Antoine Kahn

Ric Kaner

Susumu Kitagawa

Anna Koehler

Frederik Krebs

Katharina Landfester

Guglielmo Lanzani

Yan Li

Neng Li

Darren Lipomi

Bin Liu

Maria Antonietta Loi

Lynn Yueh Lin Loo

\author{
Max Planck Institute of Colloids and Interfaces \\ University of Mons \\ Carnegie Mellon University \\ Jawaharlal Nehru Centre for Advanced Scientific Research \\ Max Planck Institute for Polymer Research \\ Max Planck Institute for Polymer Research \\ University of Arizona \\ Massachusetts Institute of Technology \\ University of Alberta \\ South China University of Technology \\ Tianjin University \\ RMIT University \\ University of Cambridge \\ Soochow University \\ University of Washington \\ IISER Kolkata \\ University of Strasbourg \\ Vienna University of Technology \\ Massachusetts Institute of Technology \\ Technion - Israel Institute of Technology \\ University of Cambridge \\ Jawaharlal Nehru Centre for Advanced Scientific Research \\ Hokkaido University \\ University of California, Berkeley \\ University of Warwick \\ Imperial College London \\ University of Twente \\ Kyoto University \\ Princeton University \\ UCLA \\ Kyoto University and iCeMS \\ University of Bayreuth \\ Elite Sciences \\ Max Planck Institute for Polymer Research \\ Italian Institute of Technology \\ Peking University \\ Wuhan University of Technology \\ University of California, San Diego \\ National University of Singapore \\ University of Groningen \\ Princeton University
}

Germany

Belgium

USA

India

Germany

Germany

USA

USA

Canada

China

China

Australia

UK

China

USA

India

France

Austria

USA

Israel

UK

India

Japan

USA

UK

UK

The Netherlands

Japan

USA

USA

Japan

Germany

Denmark

Germany

Italy

China

China

USA

Singapore

Netherlands

USA 
Bettina Lotsch

HongYee Low

Eva Malmström Jonsson

Uttam Manna

Richard Martel

Hedi Mattoussi

David Mecerreyes

Phillip Messersmith

Catherine Murphy

KS Narayan

Markus Niederberger

Teri Odom

Wee-Jun Ong

Moon Jeong Park

Marie-Paule Pileni

Vivek Polshettiwar

C.N.R. Rao

Erin Ratcliff

Vince Rotello

Christine Schmidt

Gregory Scholes

Rachel Segalman

Peter Skabara

Henry Snaith

Takao Someya

Kazuo Takimiya

Luisa Torsi

Aleks Vojvodic

Elizabeth von Hauff

Shu Wang

Tanja Weil

Emily Weiss

David Weitz

Christopher M. Wolverton

Yi Xie

Vivian Wing-Wah Yam

Shannon Yee

Shu-Hong Yu

Jihong Yu

Aldo Zarbin

Xiaowei Zhan

Dongyuan Zhao
Max Planck Institute for Solid State Research

Singapore University of Technology and Design

KTH Royal Institute of Technology

Indian Institute of Technology Guwahati

University of Montreal

Florida State University

University of the Basque Country

Northwestern University

University of Illinois Urbana-Champaign

Jawaharlal Nehru Centre for Advanced Scientific Research

Swiss Federal Institute of Technology Zurich

Northwestern University

Xiamen University

Pohang University of Science and Technology

University Pierre \& Marie Curie

Tata Institute of Fundamental Research

Jawaharlal Nehru Centre for Advanced Scientific Research

University of Arizona

University of Massachusetts, Amherst

University of Florida

Princeton University

University of California Santa Barbara

University of Strathclyde

University of Oxford

University of Tokyo

RIKEN

University of Bari

University of Pennsylvania

VU Amsterdam

Institute of Chemistry, Chinese Academy of Sciences

Max Planck Institute for Polymer Research

Northwestern University

Harvard University

Northwestern University

University of Science and Technology of China

University of Hong Kong

Georgia Institute of Technology

USTC Hefei

Jilin University

Universidade Federal do Paraná

Peking University

Fudan University
Germany

Singapore

Sweden

India

Canada

USA

Spain

USA

USA

India

Switzerland

USA

Malaysia

South Korea

France

India

India

USA

USA

USA

USA

USA

UK

UK

Japan

Japan

Italy

USA

The Netherlands

China

Germany

USA

USA

USA

China

China

USA

China

China

Brazil

China

China

Seth Marder, Editor-in-Chief 